\title{
ENSINO REMOTO EMERGENCIAL: INVESTIGAÇÃO DOS FATORES DE APRENDIZADO NA EDUCAÇÃO SUPERIOR
}

\author{
ENSEÑANZA REMOTA DE EMERGENCIA: INVESTIGACIÓN DE LOS FACTORES \\ DE APRENDIZAJE EN LA ENSEÑANZA SUPERIOR
}

\author{
EMERGENCY REMOTE TEACHING: INVESTIGATION OF LEARNING FACTORS \\ IN HIGHER EDUCATION
}

\author{
Nicole Cecchele LAGO ${ }^{1}$ \\ Stela Xavier TERRA ${ }^{2}$ \\ Carla Schwengber ten CATEN ${ }^{3}$ \\ José Luis Duarte RIBEIRO ${ }^{4}$
}

RESUMO: Diante da pandemia do Coronavírus, o ensino remoto emergencial foi adotado por grande parte das instituições de ensino superior no mundo. Considerando as súbitas transformações e desafios nesse contexto, o artigo teve por objetivo identificar fatores importantes para o aprendizado no período de ensino remoto. Para tanto, um estudo quantitativo com fins explicativos foi conduzido. A questão de pesquisa foi investigada por meio de um experimento fatorial, realizado com 1069 alunos de graduação de uma universidade brasileira através de um questionário online. A análise de variância foi utilizada para avaliar o efeito dos fatores no aprendizado dos estudantes durante o período remoto. As descobertas revelam que as aulas diretas contribuem mais para o aprendizado dos alunos. Ainda, há uma leve preferência pela dinâmica de trabalho em grupos e pelo uso da plataforma Google Meet, sendo esta preferência maior para os alunos de hard-science. Especificamente, o estudo fornece orientações aos gestores universitários em direção à implementação das práticas mais favoráveis para o aprendizado durante o ensino remoto. Além disso, as descobertas podem auxiliar o desenvolvimento de estratégias para a criação de modelos educacionais resilientes e sustentáveis, baseados em práticas que potencializam o aprendizado dos alunos.

PALAVRAS-CHAVE: Educação superior. Ensino remoto emergencial. Fatores de aprendizagem. COVID-19.

\footnotetext{
${ }^{1}$ Universidade Federal do Rio Grande do Sul (UFRGS), Porto Alegre - RS - Brasil. Mestranda no Programa de Pós-Graduação em Engenharia de Produção e Transportes. ORCID: https://orcid.org/0000-0003-1523-6713. Email: nicole.cecchele@ufrgs.br

${ }^{2}$ Universidade Federal do Rio Grande do Sul (UFRGS), Porto Alegre - RS - Brasil. Mestranda no Programa de Pós-Graduação em Engenharia de Produção e Transportes. ORCID: https://orcid.org/0000-0002-5395-9667. Email: stela.xavier.terra@gmail.com

${ }^{3}$ Universidade Federal do Rio Grande do Sul (UFRGS), Porto Alegre - RS - Brasil. Professora Titular no Programa de Pós-Graduação em Engenharia de Produção e Transportes. Doutorado em Engenharia de Minas, Metalúrgica e de Materiais (UFRGS). Bolsista de Produtividade em Pesquisa do CNPq - Nível 2. ORCID: https://orcid.org/0000-0002-7904-0974. E-mail: carlacaten@gmail.com

${ }^{4}$ Universidade Federal do Rio Grande do Sul (UFRGS), Porto Alegre - RS - Brasil. Professor Titular no Programa de Pós-Graduação em Engenharia de Produção e Transportes. Doutorado em Engenharia Civil (UFRGS). Bolsista de Produtividade em Pesquisa do CNPq - Nível 1A. ORCID: https://orcid.org/0000-0002-5795-4468. E-mail: ribeiro@producao.ufrgs.br
} 
RESUMEN: La propagación del Coronavirus obligó a las autoridades educativas a implementar medidas de emergencia para combatir la discontinuidad del aprendizaje y la propagación del virus. En este sentido, la mayoría de las instituciones de educación superior del mundo está adoptando la educación remota de emergencia. Teniendo en cuenta los cambios repentinos y los desafios en este contexto, este trabajo tuvo como objetivo identificar factores importantes para este proceso de aprendizaje a distancia. Para eso, se empleó un estudio cuantitativo con fines explicativos. La pregunta de investigación fue investigada a través de un experimento factorial, realizado con 1069 estudiantes de pregrado de una universidad brasileña a través de un instrumento en línea. Se utilizó el análisis de varianza para evaluar el efecto de los factores en el aprendizaje de los estudiantes durante el período remoto. Los hallazgos revelan que las lecciones directas contribuyen más al aprendizaje de los estudiantes, independientemente de otros factores. Además, existe una dinámica de trabajo en grupo de preferencia y la plataforma Google Meet. Estas preferencias han demostrado ser más altas para los estudiantes de ciencias duras. Los resultados pueden orientar a los directores universitarios para implementar las prácticas de aprendizaje más favorables en una situación de aprendizaje a distancia. Además, pueden ayudar a desarrollar estrategias para crear modelos educativos resistentes y sostenibles basados en prácticas que mejoran el aprendizaje de los estudiantes.

$\boldsymbol{P A L A B R A S ~ C L A V E : ~ E d u c a c i o ́ n ~ u n i v e r s i t a r i a . ~ E n s e n ̃ a n z a ~ r e m o t a ~ d e ~ e m e r g e n c i a . ~ F a c t o r e s ~ d e ~}$ aprendizaje. COVID-19.

ABSTRACT: Coronavirus's spread forced educational authorities to implement emergency measures to combat the discontinuity of learning and spread the virus. In this sense, most institutions of higher education in the world are adopting emergency remote education. Based on the sudden changes and challenges in this context, this paper aimed to identify important factors for this remote learning process. For that, a quantitative study for explanatory purposes was employed. The research question was investigated through a factorial experiment, carried out with 1069 undergraduate students from a Brazilian university through an online instrument. Analysis of variance was used to assess the effect of factors on student learning during the remote period. Findings reveal direct lessons contribute more to students' learning, regardless of other factors. Also, there is a preference for group work dynamics and the Google Meet platform. Such preferences are higher for hard-science students. Results may guide university managers towards implementing the most favorable learning practices in a remote learning situation. Besides, they may help develop strategies for creating resilient and sustainable educational models based on practices that enhance students' learning.

KEYWORDS: Higher education. Emergency remote teaching. Learning factors. COVID-19.

\section{Introdução}

Devido ao surto de COVID-19 em escala mundial, as instituições acadêmicas foram obrigadas a cancelar totalmente o ensino presencial. Nesse cenário, o setor global de E Superior, assim como outros setores industriais, foi extremamente afetado pela pandemia do Coronavírus. Dessa forma, autoridades educacionais de diversos países em conjunto a especialistas em 
educação passaram a discutir a utilização da tecnologia da informação e da comunicação (TIC) para reformar o sistema educacional universitário em meio ao contexto pandêmico, a partir da oferta de ensino remoto e online (HUANG et al., 2020).

No Brasil, o Ministério da Educação (MEC) criou um website para atualizar o status de funcionamento das Instituições Federais de Ensino (IFEs). Dados referentes ao mês de agosto de 2020 mostram que aproximadamente 650 mil discentes estavam com as aulas totalmente suspensas em 42 IFEs, os quais perfazem $57,8 \%$ de uma população de aproximadamente 1,1 milhão de discentes. Analogamente, em torno de 475 mil discentes de 27 IFEs seguiam com aulas em formato remoto (BRASIL, 2020).

De fato, as medidas emergenciais adotadas são úteis para minimizar o impacto no sistema de educação. No entanto, inúmeros desafios estão associados à rápida reforma dos currículos de instituições de Educação Superior. Especificamente, esses desafios estão relacionados à preparação inadequada das universidades, cujas preocupações, segundo Mohmmed et al. (2020), limitaram-se à migração do ambiente físico para o online, ao invés de estarem voltadas à essência do ensino e à qualidade do aprendizado. Estes autores destacam que o ensino remoto ultrapassa essa migração emergencial, uma vez que é responsável pela exploração eficiente das ferramentas de ensino disponíveis no ambiente online para cumprir o currículo educacional que, em situações normais, seriam entregues fisicamente. Bittencourt, Ferraz e Mercado (2019) destacam que o ensino e a aprendizagem exercidos fora das paredes da universidade tornaram-se viáveis pelos recentes avanços nas TIC, as quais permitem conexões sociais e profissionais, mesmo quando os indivíduos estão separados pela distância geográfica (KNIGHT, 2020).

Considerando que os agentes propiciadores dessa transformação nos ambientes de ensino são os professores da Educação Superior, responsáveis por conduzir o aprendizado online, Kapasia et al. (2020) sugerem que as diretrizes para o desenvolvimento de capacidades sejam absorvidas, primeiramente, por eles. Dessa forma, dada a falta de evidências empíricas e a necessidade de conhecimento acerca dos processos de ensino e aprendizagem digitais (TESAR, 2020), uma pesquisa com estudantes de graduação de uma universidade brasileira foi conduzida, tendo por objetivo responder a seguinte questão de pesquisa: quais são os fatores importantes para o aprendizado na modalidade de ensino remoto?

Mais especificamente, a pesquisa questionou a percepção de importância de atributos investigados em diferentes cenários para identificar a relação dos fatores com o aprendizado. Metodologicamente, o estudo é caracterizado por uma abordagem quantitativa e possui finalidade explicativa, na qual a preocupação central é identificar os fatores que contribuem 
para a ocorrência dos fenômenos (GIL, 2019). Quanto ao procedimento, trata-se de um experimento fatorial realizado por meio de um questionário online com os estudantes (quais?). Os resultados foram analisados por meio da análise de variância, em que o efeito causado pela variação do nível dos fatores na percepção de importância para o aprendizado foi avaliado.

Além dos dados informados pelo MEC (2020) apresentados anteriormente, a urgência das respostas para a questão de pesquisa é evidenciada pelos achados de Kapasia et al. (2020), os quais indicam que $76 \%$ dos estudantes da Educação Superior entrevistados da Índia, cujas aulas estão sendo ministradas de forma remota, acreditam que a pandemia possa causar a interrupção dos seus estudos, caso o ensino continue nesse formato nos próximos meses. Tais circunstâncias caóticas trazem luz à necessidade de mudanças significativas a serem conduzidas pelas universidades que estabeleceram o ensino remoto (TESAR, 2020), tanto para combater a interrupção do setor quanto para tornar os sistemas educacionais resilientes (HUANG et al., 2020; KAPASIA et al., 2020).

Nesse sentido, os resultados deste estudo podem orientar os gestores e professores das universidades com relação à implementação das práticas mais favoráveis para o aprendizado em meio ao período de ensino remoto. Além disso, as descobertas podem auxiliar o desenvolvimento de estratégias para a criação de modelos educacionais sustentáveis a longo prazo, baseados em práticas de qualidade que potencializam o aprendizado dos estudantes.

\section{Planejamento experimental}

Para responder a questão de pesquisa, um planejamento experimental foi conduzido, o qual permite estudar os efeitos isolados dos fatores investigados, assim como os efeitos combinados de dois ou mais fatores (MALHOTRA, 2019). Isto é, a partir de um projeto fatorial $2^{\wedge} \mathrm{k}$ completo, cinco fatores foram investigados em dois níveis para verificar se a alteração em seus níveis provoca diferenças significativas na variável resposta, neste caso, definida como a importância para o aprendizado no período de ensino remoto. Assim, os fatores controláveis a serem alterados no experimento encontram-se na Tabela 1, com seus respectivos níveis de investigação. 
Tabela 1 - Fatores controláveis no experimento e respectivos intervalos de investigação

\begin{tabular}{lll}
\hline Fatores controláveis & Nível inferior (-1) & Nível superior (+1) \\
\hline Abordagem da aula & Direta & Invertida \\
Plataforma & Google Meet & Zoom \\
Dinâmica das atividades & Individual & Em grupo \\
Gênero & Feminino & Masculino \\
Natureza do curso & Hard & Soft \\
\hline
\end{tabular}

Fonte: Elaborado pelos autores

O primeiro fator investigado incide sobre as metodologias de aprendizagem, as quais, com a propagação da COVID-19 aliada ao uso intensificado das TIC, estão fortemente voltadas às abordagens ativas de aprendizagem (HUANG et al., 2020). De acordo com Bacich e Moran (2018), as metodologias ativas suportam uma educação inovadora que possibilita transformar aulas em experiências de aprendizagem mais significativas. Dentro desse panorama, a aula invertida é um dos métodos ativos de aprendizagem, onde o aluno possui contato prévio com o conteúdo e a aula torna-se o lugar de aprendizagem aberta, com perguntas, discussões e soluções de problemas em grupos. Assim, o professor se comporta como um orientador do aprendizado, visto que este é cocriado a partir dele e dos alunos (LIPOMI, 2020). Por outro lado, nas aulas diretas o professor transmite a informação ao aluno, que, após a aula, deve estudar o conteúdo exposto e realizar exercícios de fixação para assimilar o conteúdo (BACICH; MORAN, 2018).

A plataforma de comunicação também tem sido discutida e compreende o segundo fator do planejamento experimental. Moreira, Henriques e Barros (2020) afirmam que no contexto atual os professores foram impelidos à gravação de vídeo aulas. Isso ocorre através do sistema de videoconferência Zoom e das plataformas de aprendizagem Moodle, Microsoft Teams e Google Meet (LIPOMI, 2020; MOHMMED et al., 2020). Embora a literatura aborde muitas plataformas, apenas as ferramentas Google Meet e Zoom foram selecionadas por apresentarem maior afinidade entre os estudantes.

Outro aspecto importante para o ensino remoto é a dinâmica da aula, a qual, devido à transição do ambiente físico para o digital associada aos impactos emocionais e sociais causados pela pandemia, exigem mudanças nas práticas de sala de aula. Com isso, sugere-se que as tarefas coletivas e avaliação por pares tornem-se padrão para a redução da carga de trabalho acadêmica (TESAR, 2020). Isso corrobora as descobertas de Knight (2020), relacionadas à priorização das interações em grupos para o sucesso do aprendizado online. 
Além disso, considerando os dados do INEP (2019) relacionados à predominância do gênero feminino em instituições públicas e privadas de Educação Superior no Brasil, representada por aproximadamente $57 \%$, e, tendo por referência que os cursos de ensino e cuidado (enfermagem, nutrição, odontologia e fisioterapia, por exemplo) são ocupados, em sua maioria, por mulheres (VARGAS, 2014), optou-se por incluir o gênero como fator controlável do experimento, tendo em vista verificar se existem diferenças significativas entre os estudantes do gênero masculino e feminino.

Por fim, baseado no estudo de Parpala et al. (2010), o qual identificou diferenças significativas nas abordagens adotadas pelos alunos de cursos de graduação compreendidos em soft-science e hard-science em direção à aprendizagem, optou-se por verificar se possíveis diferenças nas percepções de importância de fatores para o ensino remoto também são existentes entre esses grupos. Hard-science, por exemplo, Matemática, Engenharia e Física, possuem um único paradigma que permite que os acadêmicos cheguem a um amplo consenso sobre métodos de pesquisa e conceitos-chave. Em contrapartida, soft-science dissertam sobre diversos métodos e conceitos-chave, compreendendo áreas de natureza social, como Direito, Filosofia e Educação. Apesar desta divisão entre as áreas de conhecimento não contemplar totalmente a complexidade das disciplinas, Dang (2018) afirma que a distinção é útil para explicar a diversidade do meio acadêmico. Por esse motivo, os estudantes foram divididos em dois grupos, de acordo com seu curso de graduação, constituindo, assim, o quinto fator controlável do experimento.

Além dos fatores controláveis, foram definidos fatores que permaneceram constantes no experimento, os quais não foram investigados, uma vez que precisavam ser mantidos em um nível fixo para não inflacionar o termo do erro ou a variabilidade experimental (MONTGOMERY, 2017). Nesse sentido, o tempo de duração da aula foi fixado em 3 horas para que todos os estudantes pudessem avaliar os cenários tendo por referência o mesmo parâmetro. Da mesma forma, considerando o estudo de Mohmmed et al. (2020), cujos resultados indicam diferenças nas percepções de alunos de graduação e pós-graduação, optouse por realizar o experimento apenas com estudantes de graduação.

\section{Procedimentos de coleta e análise de dados}

Os dados foram coletados entre os dias 26 de junho e 26 de julho de 2020 . Nesse período, os graduandos da universidade em estudo estavam próximo do encerramento do primeiro semestre letivo, embora alguns cursos de graduação tenham iniciado as aulas de forma 
parcialmente, após a Portaria $\mathrm{N}^{\mathrm{o}} 343$ do MEC, divulgada em 17 de março de 2020 (BRASIL, 2020).

A coleta de dados foi composta por dois blocos, sendo o primeiro voltado ao projeto experimental e o segundo correspondente à caracterização dos estudantes. O primeiro bloco contemplou um projeto fatorial $2^{\wedge} \mathrm{k}$, através do qual foram investigados três fatores em dois níveis, tendo em vista identificar os fatores que maximizam a variável resposta, percepção de importância para o aprendizado, por meio dos oito cenários propostos. Estes foram avaliados a partir de uma escala Likert de 7 pontos, em que a pontuação 1 significa "pouco importante", enquanto, 7 corresponde a "muito importante". Por sua vez, o segundo bloco contemplou questões de caraterização dos respondentes, relacionadas ao gênero, idade e ao curso.

O instrumento foi enviado aos estudantes de graduação da universidade por meio de $e$ mails. Assim, 1.113 respostas foram coletadas. No entanto, ao observar inconsistências no preenchimento de algumas questões, 44 respostas, (4\%) tornaram-se inviáveis para a análise dos dados. Dessa forma, o conjunto de dados reúne 1.069 respostas válidas. Destas, a maioria dos respondentes é do gênero feminino (59,59\%), a idade média é 24 anos, sendo que apenas $29,37 \%$ possuem idade superior a esta, e $70,63 \%$ possui 24 anos ou menos. Com relação à natureza dos cursos em que os estudantes estão matriculados, $64,45 \%$ pertencem ao grupo atribuído como hard-science, enquanto $35,55 \%$ se encaixam no grupo classificado como softscience. A Tabela 2 sumariza essas variáveis categóricas.

Tabela 2 - Caracterização dos respondentes

\begin{tabular}{llllll}
\hline \multicolumn{7}{c}{ Perfil dos respondentes } \\
\hline Gênero & $\mathbf{N}$ & $\mathbf{\%}$ & Faixa etária & $\mathbf{n}$ & $\%$ \\
Feminino & 637 & $59,59 \%$ & 24 anos ou menos & 755 & $70,63 \%$ \\
Masculino & 432 & $40,41 \%$ & Acima de 24 anos & 314 & $29,37 \%$ \\
\hline Total & 1069 & $100 \%$ & Total & 1069 & $100 \%$ \\
\hline Natureza do curso & $\mathbf{N}$ & $\mathbf{\%}$ & & & \\
Hard-science & 689 & $64,45 \%$ & & \\
Soft-science & 380 & $35,55 \%$ & & \\
\hline Total & 1069 & $100 \%$ & & & \\
\hline
\end{tabular}

Fonte: Elaborado pelos autores

A análise dos dados foi realizada por meio do software estatístico Minitab versão 19. Inicialmente, a análise de confiabilidade das variáveis do instrumento foi conduzida através do Alfa de Cronbach para o bloco dos cenários. A Tabela 3 apresenta os resultados. 
Tabela 3 - Teste de confiabilidade das variáveis

\begin{tabular}{ccc}
\hline Alpha de Cronbach & Alpha de Cronbach baseado na padronização dos itens & Número de itens \\
\hline 0,7540 & 0,7508 & 8
\end{tabular}

Fonte: Elaborado pelos autores

A Tabela 3 mostra que o resultado para o primeiro bloco obteve valor superior a 0,7 , fornecendo evidências de confiabilidade e apresentando um ótimo nível de consistência interna, conforme os parâmetros recomendados por Malhotra (2019). Na sequência, a matriz experimental foi realizada a partir da média ponderada para cada um dos cenários, a fim de definir a variável resposta correspondente. A Tabela 4 apresenta a matriz dos fatores do experimento fatorial completo $2^{\wedge} 5$ em níveis reais investigados e as respectivas percepções de importância dos estudantes. É válido notar que todos os efeitos principais e interações de dois fatores foram testadas, ao contrário das interações compreendendo três ou mais fatores, uma vez que estas foram utilizadas para estimar o termo do erro (MONTGOMERY, 2017).

Posteriormente, utilizou-se estatística descritiva para analisar as características demográficas dos estudantes. Finalmente, para as questões que avaliaram a percepção de importância dos estudantes com base em cenários, foram realizadas análises de variância (ANOVA), para testar se uma variação entre os níveis dos fatores controláveis definidos representava uma diferença significativa na variável resposta, ou seja, na percepção de importância com relação ao aprendizado em meio ao contexto de ensino remoto.

Tabela 4 - Definição da matriz experimental

\begin{tabular}{ccccccc}
\hline Cenário & Fator X1 & Fator X2 & Fator X3 & Fator X4 & Fator X5 & VR \\
\hline 1 & Direta & Google Meet & Individual & Feminino & Hard & 4,731 \\
2 & Invertida & Google Meet & Individual & Feminino & Hard & 3,522 \\
3 & Direta & Zoom & Individual & Feminino & Hard & 4,678 \\
4 & Invertida & Zoom & Individual & Feminino & Hard & 3,386 \\
5 & Direta & Google Meet & Em grupo & Feminino & Hard & 4,974 \\
6 & Invertida & Google Meet & Em grupo & Feminino & Hard & 3,609 \\
7 & Direta & Zoom & Em grupo & Feminino & Hard & 4,828 \\
8 & Invertida & Zoom & Em grupo & Feminino & Hard & 3,452 \\
9 & Direta & Google Meet & Individual & Masculino & Hard & 4,758 \\
10 & Invertida & Google Meet & Individual & Masculino & Hard & 3,515 \\
11 & Direta & Zoom & Individual & Masculino & Hard & 4,811 \\
12 & Invertida & Zoom & Individual & Masculino & Hard & 3,424 \\
13 & Direta & Google Meet & Em grupo & Masculino & Hard & 4,947 \\
14 & Invertida & Google Meet & Em grupo & Masculino & Hard & 3,571 \\
15 & Direta & Zoom & Em grupo & Masculino & Hard & 4,875 \\
\hline
\end{tabular}




\begin{tabular}{|c|c|c|c|c|c|}
\hline Invertida & Zoom & Em grupo & Masculino & Hard & 3,428 \\
\hline Direta & Google Meet & Individual & Feminino & Soft & 4,774 \\
\hline Invertida & Google Meet & Individual & Feminino & Soft & 3,434 \\
\hline $\begin{array}{c}\text { Direta } \\
\text { Invertida }\end{array}$ & $\begin{array}{l}\text { Zoom } \\
\text { Zoom }\end{array}$ & $\begin{array}{l}\text { Individual } \\
\text { Individual }\end{array}$ & $\begin{array}{l}\text { Feminino } \\
\text { Feminino }\end{array}$ & $\begin{array}{l}\text { Soft } \\
\text { Soft }\end{array}$ & $\begin{array}{l}4,854 \\
3,401\end{array}$ \\
\hline Direta & Google Meet & Em grupo & Feminino & Soft & 4,910 \\
\hline Invertida & Google Meet & Em grupo & Feminino & Soft & 3,519 \\
\hline Direta & Zoom & Em grupo & Feminino & Soft & 4,868 \\
\hline Invertida & Zoom & Em grupo & Feminino & Soft & 3,443 \\
\hline Direta & Google Meet & Individual & Masculino & Soft & 4,708 \\
\hline Invertida & Google Meet & Individual & Masculino & Soft & 3,382 \\
\hline Direta & Zoom & Individual & Masculino & Soft & 4,780 \\
\hline Invertida & Zoom & Individual & Masculino & Soft & 3,375 \\
\hline Direta & Google Meet & Em grupo & Masculino & Soft & 4,863 \\
\hline Invertida & Google Meet & Em grupo & Masculino & Soft & 3,488 \\
\hline Direta & Zoom & Em grupo & Masculino & Soft & 4,792 \\
\hline Invertida & Zoom & Em grupo & Masculino & Soft & 3,405 \\
\hline
\end{tabular}

Fonte: Elaborado pelos autores

\section{Análise e discussão dos resultados}

A ANOVA indica que existe uma relação estatisticamente significativa entre as variáveis ao nível de confiança de $95 \%$ e valor-p $<0,05$, conforme apresentado na Tabela 5 .

Tabela 5 - Resultados da análise de variância (ANOVA)

\begin{tabular}{llllll}
\hline Fonte & GL & SQ (Aj.) & QM $(\mathbf{A j .}$ ) & Valor F & Valor-P \\
\hline Modelo & 15 & 15,009 & 1,001 & 958,33 & 0,000 \\
Linear & 5 & 14,946 & 2,989 & 2863,05 & 0,000 \\
Aula & 1 & 14,845 & 14,845 & 14218,28 & $\mathbf{0 , 0 0 0}$ \\
Plataforma & 1 & 0,026 & 0,026 & 24,67 & $\mathbf{0 , 0 0 0}$ \\
Dinâmica & 1 & 0,065 & 0,065 & 62,25 & $\mathbf{0 , 0 0 0}$ \\
Gênero & 1 & 0,002 & 0,002 & 2,07 & 0,170 \\
Curso & 1 & 0,008 & 0,008 & 7,98 & $\mathbf{0 , 0 1 2}$ \\
Interações de 2 fatores & 10 & 0,062 & 0,006 & 5,97 & 0,001 \\
Aula*Plataforma & 1 & 0,009 & 0,009 & 8,91 & $\mathbf{0 , 0 0 9}$ \\
Aula*Dinâmica & 1 & 0,007 & 0,007 & 7,08 & $\mathbf{0 , 0 1 7}$ \\
Aula*Gênero & 1 & 0,000 & 0,000 & 0,26 & 0,614 \\
Aula*Curso & 1 & 0,005 & 0,005 & 5,02 & $\mathbf{0 , 0 4 0}$ \\
Plataforma*Dinâmica & 1 & 0,014 & 0,014 & 13,69 & $\mathbf{0 , 0 0 2}$ \\
Plataforma*Gênero & 1 & 0,002 & 0,002 & 1,47 & 0,243 \\
Plataforma*Curso & 1 & 0,011 & 0,012 & 10,34 & $\mathbf{0 , 0 0 5}$ \\
Dinâmica*Gênero & 1 & 0,001 & 0,001 & 1,27 & 0,276 \\
Dinâmica*Curso & 1 & 0,002 & 0,002 & 2,32 & 0,147 \\
Gênero*Curso & 1 & 0,010 & 0,0010 & 9,37 & $\mathbf{0 , 0 0 7}$
\end{tabular}




\begin{tabular}{lllll} 
Erro & 16 & 0,017 & 0,001 & \\
Total & 31 & 15,026 & & \\
$\mathbf{R}^{2}$ & $99,89 \%$ & & $\mathbf{S}$ & 0,032 \\
$\mathbf{R}^{2}$ ajustado & $99,78 \%$ & & & \\
\hline
\end{tabular}

Fonte: Elaborado pelos autores

Os resultados mostram que há uma relação entre o aprendizado com quatro dos efeitos principais e com seis interações. O coeficiente $\mathrm{R}^{2}$ indica que o modelo explica 99,89\% da variabilidade da percepção de importância dos fatores investigados com relação ao aprendizado. Nesse sentido, ao analisar os efeitos principais, é possível observar que a percepção de importância varia, significativamente, de acordo com a abordagem da aula, a plataforma em que a aula é ministrada, a dinâmica estabelecida pelo professor e a classificação do curso do estudante. Mais especificamente, esses fatores influenciam a percepção de importância dos fatores de ensino remoto, estatisticamente, ao nível de significância $\alpha=0,05$. Ou seja, o gênero analisado de forma isolada não é responsável por alterar a percepção de importância avaliada pelos estudantes. A Figura 1 apresenta os gráficos dos efeitos principais para os fatores que apresentaram significância estatística.

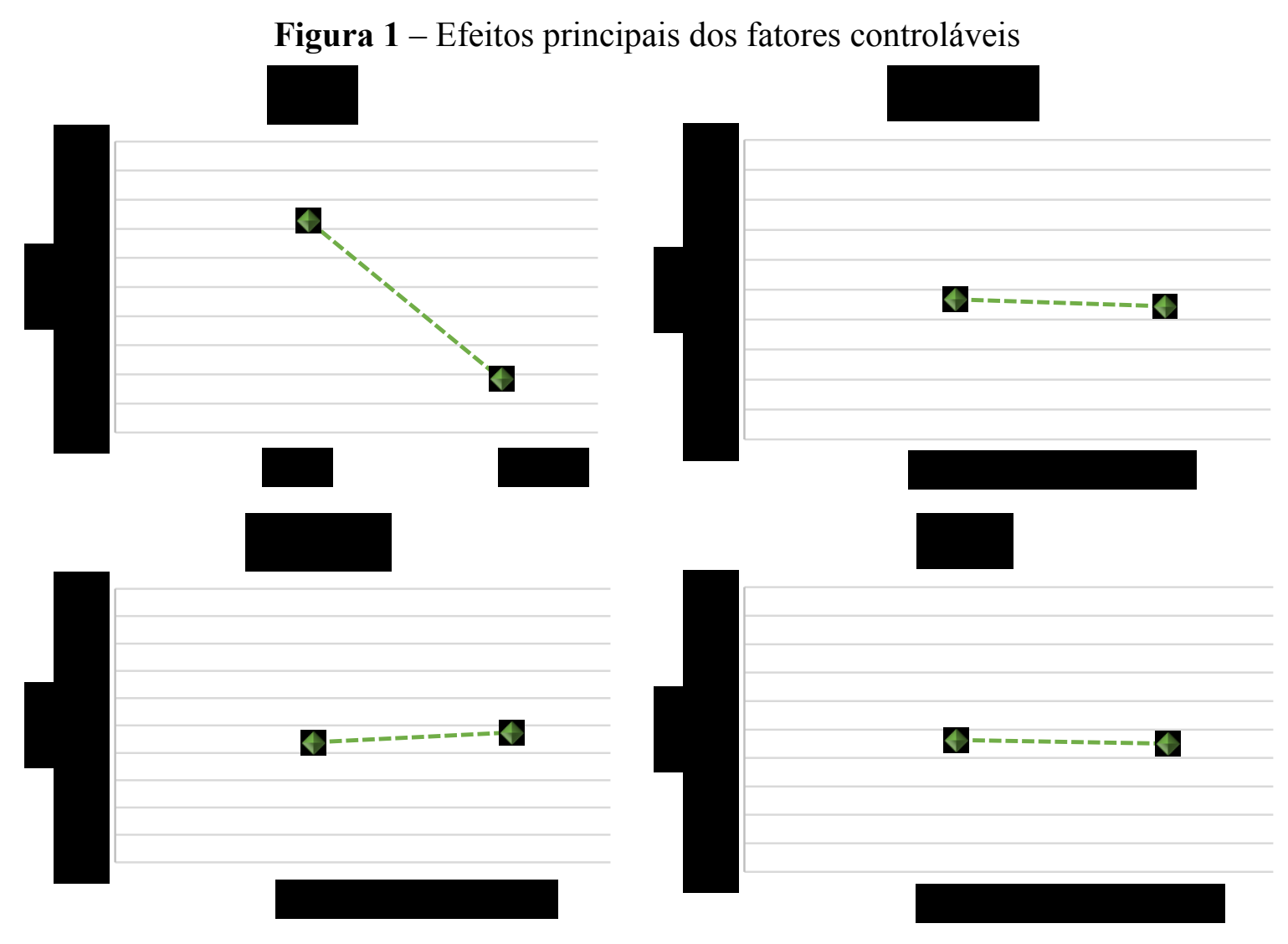

Fonte: Elaborado pelos autores 
Os gráficos dos efeitos principais mostram que os estudantes destinaram pontuações mais altas aos cenários em que a aula é ministrada de forma direta, ou seja, aulas diretas contribuem mais para o aprendizado do que aulas invertidas. Isso corrobora com os achados de Knight (2020) e Mohmmed et al. (2020), de que o método direto é mais eficaz para o aprendizado do que o ensino invertido. No entanto, as descobertas contrastam com as recomendações de Huang et al. (2020), os quais afirmam que o ensino remoto emergencial exige maior envolvimento dos alunos e, portanto, sugerem que o ensino invertido seja implementado para incentivar os alunos a participarem da cocriação do conhecimento.

Para a plataforma, há uma leve preferência pela Google Meet em comparação ao Zoom, o que corrobora as sugestões de Kapasia et al. (2020). Houve também uma leve preferência pela dinâmica da aula conduzida em grupos em comparação aos trabalhos realizados de forma individual. Por fim, observa-se que graduandos de cursos hard-sciences atribuíram pontuações mais altas aos cenários avaliados do que os estudantes de soft-sciences. Em suma, esses quatro fatores são responsáveis por influenciar a percepção de importância dos alunos com relação ao aprendizado independentemente de outros fatores.

Com relação aos efeitos da interação de dois fatores, a Figura 2 mostra que a importância percebida pelos estudantes é alterada de acordo com a modificação dos níveis desses fatores, isto é, o efeito de um fator sobre a variável resposta depende, mesmo que levemente, do nível do segundo fator. Os resultados mostram que a aula invertida afeta mais a percepção de importância dos estudantes quando a plataforma analisada é o Zoom. Mais especificamente, embora a percepção de importância para a plataforma Zoom seja menor tanto quando a aula é direta quanto invertida, ela é ainda menor com a aula invertida. Assim, observa-se uma relação de dependência entre a abordagem da aula e a plataforma utilizada, visto que quando a abordagem da aula passa de direta para invertida, a percepção de importância dos estudantes é alterada, de acordo com o nível do segundo fator, isto é, da plataforma.

Figura 2 - Efeitos das interações significativas entre dois fatores 


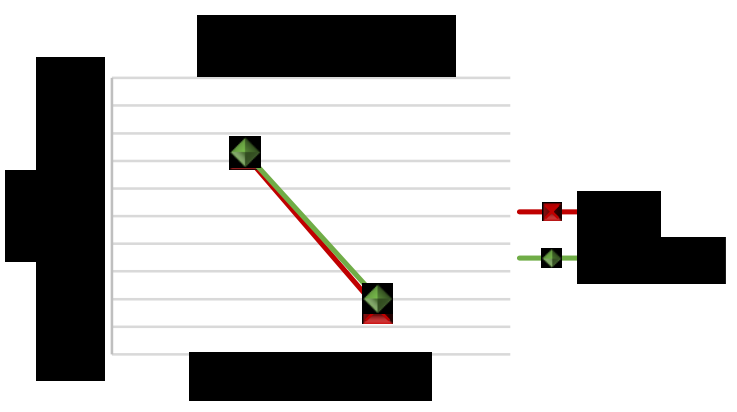

$2 \mathrm{a}$

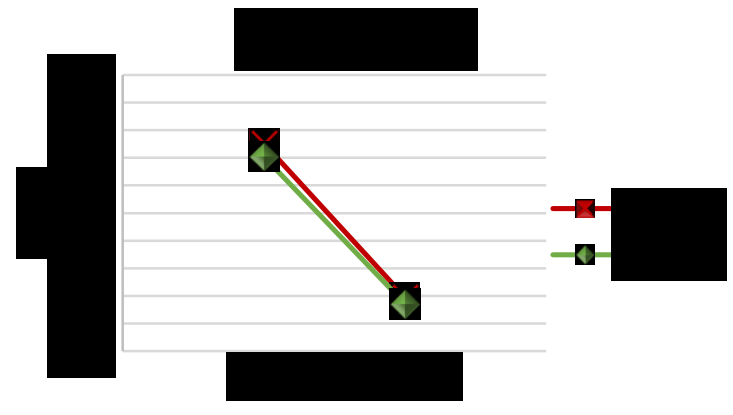

$2 \mathrm{c}$

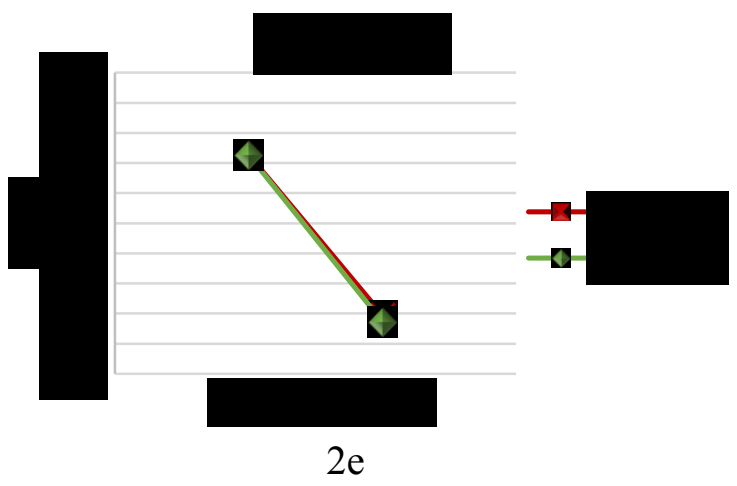

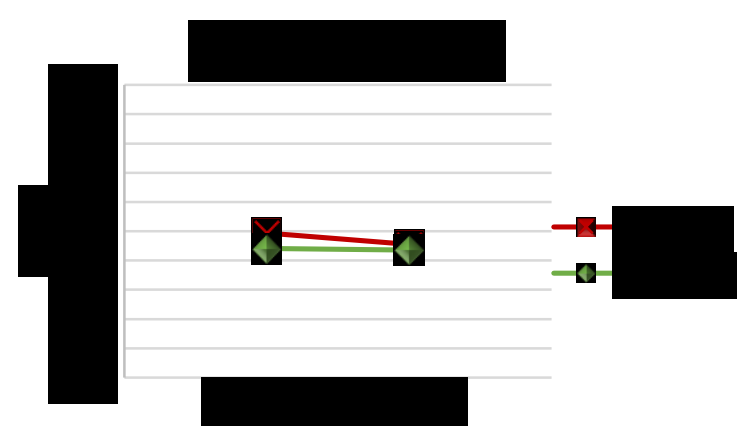

$2 b$

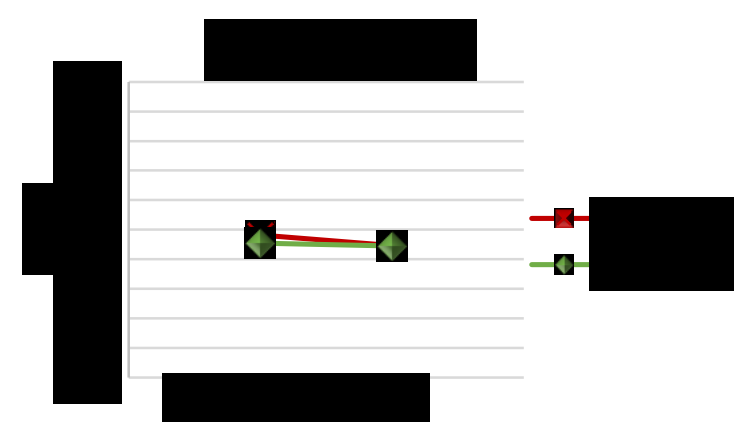

$2 d$

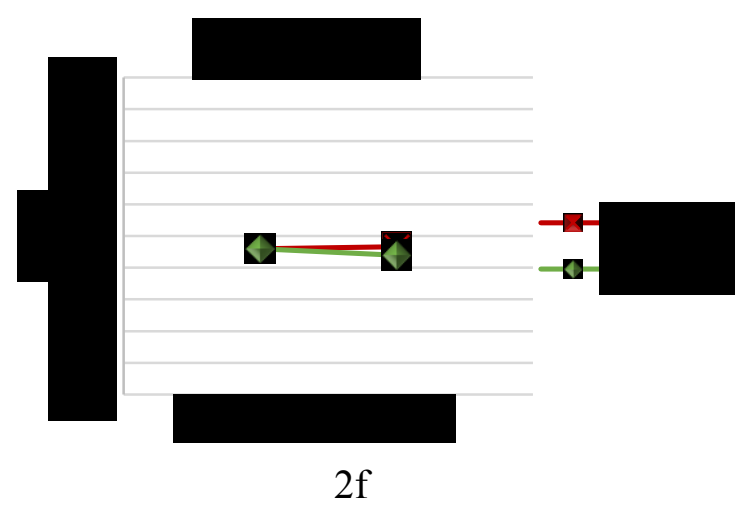

Fonte: Elaborado pelos autores

O mesmo ocorre na interação entre a aula e a dinâmica, visto que quando a aula é do tipo direta ou mesmo do tipo invertida, a percepção de importância dos alunos é mais alta quando a dinâmica é em grupo do que quando é de forma individual. Ainda, a abordagem da aula em interação com a área do curso do estudante indica que aulas diretas são percebidas como mais importantes para o aprendizado em detrimento das aulas invertidas, tanto para curso hard-science quanto soft-science. Em suma, o grau de importância aumenta substancialmente quando a aula é do tipo direta nos três fatores (plataforma, dinâmica e curso). Com relação aos níveis destes, embora as interações não tenham sido muito fortes, observa-se que a ampliação 
da percepção de importância dos estudantes ocorre nos cenários representados pela plataforma Google Meet, dinâmica em grupo e cursos hard-science.

$\mathrm{Na}$ interação entre plataforma e dinâmica, os resultados revelam que quando a plataforma varia do Zoom para o Google Meet, a percepção de importância dos estudantes é maior quando a dinâmica do trabalho é em grupo. Isso contrasta com o estudo de Lipomi (2020), que relaciona a dinâmica das aulas com a utilização da plataforma Zoom, uma vez que esta fornece a possibilidade de formar grupos de interação a partir das salas virtuais (breakout rooms).

Semelhantemente ocorre para o efeito da interação entre plataforma e curso, visto que quando a mesma transição entre as plataformas acontece, os estudantes dos cursos hard-science atribuem notas mais altas do que os estudantes dos cursos soft-science. Finalmente, observa-se o efeito da interação entre o gênero e as escolhas pelos cursos hard-science e soft-science. Nesse sentido, a Figura $2 \mathrm{f}$ evidencia que o gênero masculino dos estudantes matriculados em cursos categorizados como hard-science e o gênero feminino dos cursos de soft-science atribuíram pontuações mais altas para os cenários investigados.

\section{Considerações finais}

A pandemia COVID-19 ocasionou profundas transformações na organização social e em diversos setores do mundo. Diante deste cenário, os estudos recentes voltados aos impactos do isolamento no ensino, em sua maioria, contemplam discussões pertinentes acerca das oportunidades e barreiras associadas ao novo ambiente educacional, entretanto, possuem natureza predominantemente teórica. Além disso, embora alguns estudos tenham investigado as percepções dos professores e a satisfação dos alunos com relação ao ensino remoto, os fatores que contribuem para o aprendizado dos estudantes de ensino superior permaneciam desconhecidos. Considerando essa lacuna, este estudo teve por objetivo investigar os fatores importantes para o aprendizado sob o ponto de vista dos estudantes de graduação de uma universidade brasileira durante o período de ensino remoto emergencial.

Os resultados evidenciaram que a abordagem da aula, a plataforma utilizada, a dinâmica para a execução dos trabalhos e o curso do estudante influenciam a percepção de importância dos alunos com relação ao aprendizado. Especificamente, as descobertas revelaram que os estudantes acreditam que as aulas diretas, a plataforma Google Meet e a dinâmica dos trabalhos em grupo contribuem mais para o aprendizado do que os seus níveis opostos. Ainda nesse sentido, o experimento revelou diferenças significativas entre as percepções dos estudantes 
matriculados em cursos de natureza hard-science e soft-science. Isso significa que as implicações decorrentes dos resultados não podem ser generalizadas para todos os cursos de graduação, visto que há diferenças significativas, mesmo que pequenas, entre as percepções dos estudantes dos dois grupos.

Os achados também evidenciaram que a leve preferência dos estudantes por trabalho em grupos é superior principalmente quando a plataforma Google Meet é utilizada. Da mesma forma, a percepção de importância dos estudantes para essa plataforma é levemente maior para os alunos de hard-science. Nesse sentido, tendo por referência a natureza conclusiva dos resultados, as descobertas podem ajudar pesquisadores e educadores a aprimorarem as experiências de aprendizagem dos estudantes durante o período de isolamento social ocasionado pelo surto do COVID-19. Embora o objetivo do estudo não tenha sido a geração de proposições para o contexto investigado, os resultados fornecem evidências que podem atuar como insumo para a elaboração de futuras estratégias e políticas relacionadas ao ensino remoto, tendo por objetivo melhorar o desempenho das atividades nos processos de ensino e aprendizagem em circunstâncias semelhantes.

Como limitação de pesquisa, destaca-se a execução do experimento de forma completa, ou seja, os estudantes avaliaram os oito cenários do instrumento, o que pode ter ocasionado cansaço ou fadiga no momento do preenchimento das questões. Assim, sugere-se que estudos futuros, que utilizem projetos fatoriais, realizem o processo de blocagem do experimento. Além disso, sugere-se que uma pesquisa com grupos focais investigue a preferência dos estudantes por aulas diretas, uma vez que a literatura encoraja os profissionais da educação a investirem nas metodologias ativas e assíncronas. Igualmente, futuras pesquisas poderiam ser realizadas para entender a relação entre a plataforma Google Meet e a dinâmica em grupos, uma vez que esta plataforma não oferece recursos que facilitem a separação dos estudantes em grupos, como a plataforma Zoom, por exemplo. Por fim, o estudo poderia ser replicado com alunos de pósgraduação e do ensino médio, para identificar possíveis diferenças entre as percepções dos estudantes.

AGRADECIMENTOS: Os autores agradecem à Coordenação de Aperfeiçoamento de Pessoal de Nível Superior do Brasil (CAPES) e ao Conselho Nacional de Desenvolvimento Científico e Tecnológico (CNPq) pelo apoio financeiro recebido para a realização desta pesquisa.

\section{REFERÊNCIAS}


BACICH, L.; MORAN, J. Metodologias ativas para uma educação inovadora: uma abordagem teórico-prática. Porto Alegre: Penso, 2018.

BITTENCOURT, I. M.; FERRAZ, E. S.; MERCADO, L. P. L. O uso de ferramenta síncrona na coleta de dados na pesquisa em educação online. Revista Ibero-Americana de Estudos em Educação, Araraquara, v. 14, n. 3, p. 1217-1228, jul./set. 2019. e-ISSN: 1982-5587. DOI: https://doi.org/10.21723/riaee.v14i3.9053

BRASIL. Ministério da Educação. Coronavírus. Monitoramento nas instituições de ensino. Brasília, DF, MEC, 2020. Acesso em: 10 ago. 2020. Disponível em: http://portal.mec.gov.br/coronavirus/. Acesso em: 10 dez. 2020.

DANG, T. N. Y. The nature of vocabulary in academic speech of hard and soft-sciences.

English For Specific Purposes, v. 51, p. 69-83, 2018. DOI:

https://doi.org/10.1016/j.esp.2018.03.004

GIL, A. C. Métodos e técnicas de pesquisa social. 7. ed. São Paulo: Atlas, 2019.

HUANG, R. et al. Disrupted classes, undisrupted learning during COVID-19 outbreak in China: application of open educational practices and resources. Smart Learn. Environ., v. 7, n. 1, p. 1-15, 2020. DOI: https://doi.org/10.1186/s40561-020-00125-8

INEP. Resumo Técnico do Censo da Educação Superior 2017. Brasília: Instituto Nacional de Estudos e Pesquisas Educacionais Anísio Teixeira, 2019.

KAPASIA, N. et al. Impact of lockdown on learning status of undergraduate and postgraduate students during COVID-19 pandemic in West Bengal, India. Children and Youth Services Review, 116, p. 1-5, 2020. DOI: https://doi.org/10.1016/j.childyouth.2020.105194

KNIGHT, S. W. P. Establishing professional online communities for world language educators. Foreign Language Annals, v. 53, n. 2, p. 298-305, 2020. DOI:

https://doi.org/10.1111/flan.12458

LIPOMI, D. J. Video for active and remote learning. Trends in Chemistry, v. 2, n. 6, p. 483485, 2020. DOI: https://doi.org/10.1016/j.trechm.2020.03.003

MALHOTRA, N. K. Pesquisa de marketing: uma orientação aplicada. 7. ed. Porto Alegre: Bookman, 2019.

MOHMMED, A. O. et al. Emergency remote teaching during Coronavirus pandemic: the current trend and future directive at Middle East College Oman. Innovative Infrastructure Solutions, v. 5, n. 3, p. 1-11, 2020. DOI: https://doi.org/10.1007/s41062-020-00326-7

MONTGOMERY, D. C. Design and analysis of experiments. 9. ed. Wiley: London, 2017.

MOREIRA, J. A. M.; HENRIQUES, S.; BARROS, D. Transitando de um ensino remoto emergencial para uma educação digital em rede, em tempos de pandemia. Rev. Dialogia, São Paulo, n. 34, p. 351-364, 2020. DOI: https://doi.org/10.5585/dialogia.n34.17123 
PARPALA, A. et al. Students' approaches to learning and their experiences of the teachinglearning environment in different disciplines. British Journal of Educational Psychology, v. 80, n. 2, p. 269-282, 2010. DOI: https://doi.org/10.1348/000709909x476946

TESAR, M. Towards a post-covid-19 'new normality?': physical and social distancing, the move to Online and Higher Education. Policy Futures in Education, v. 18, n. 5, p. 556-559, 2020. DOI: https://doi.org/10.1177\%2F1478210320935671

VARGAS, R. B. Haverá lugar para o olhar de gênero na ciência brasileira? Cadernos de Gênero e Tecnologia, v. 8, n. 29, p. 20-32, 2014. DOI:

http://dx.doi.org/10.3895/cgt.v8n29/30.6124

\section{Como referenciar este artigo}

LAGO, N. C.; TERRA, S. X.; CATEN, C. S.; RIBEIRO, J. L. D. Ensino Remoto Emergencial: investigação dos fatores de aprendizado na educação superior. Revista Ibero-Americana de Estudos em Educação, Araraquara, v. 16, n. 2, p. 391-406, abr./jun. 2021. e-ISSN: 1982-5587. DOI: https://doi.org/10.21723/riaee.v16i2.14439

Submetido em: 09/11/2020

Revisões requeridas em: 21/12/2020

Aprovado em: 29/01/2021

Publicado em: 01/02/2021 